\title{
Modelling Study of Deformation Texture and Plastic Heterogeneity at Grain Boundaries and Triple Junctions
}

\author{
VAN HOUTTE Paul ${ }^{1, a}$, KANJARLA Anand Krishna ${ }^{1, b}$ and DELANNAY \\ Laurent $^{2, \mathrm{c}}$ \\ ${ }^{1}$ Departement of Metallurgy and Materials Engineering, Katholieke Universiteit Leuven, \\ Kasteelpark Arenberg 44, Bus 2450, BE-3001, Leuven, Belgium \\ 2 Department of Mechanical Engineering, Université Catholique de Louvain, \\ av. G. Lemaître 4, BE-1348 Louvain-la-Neuve, Belgium \\ apaul.vanhoutte@mtm.kuleuven.be, ${ }^{\mathrm{b}}$ AnandKrishna.Kanjarla@mtm.kuleuven.be, \\ cdelannay@mema.ucl.ac.be
}

Keywords: Plastic deformation, polycrystal, texture, CPFEM, grain boundaries, triple junction, heterogeneity.

\begin{abstract}
A CPFE model was used for an assessment of the assumptions used by the ALAMEL model concerning grain interactions. A finite element mesh was constructed for a multicrystal consisting of four grains. There were 17496 integration points per grain. The main goal was to capture the complex nature of the plastic fields in the vicinity of the grain boundaries. The distribution of strain rates, both along and perpendicular to the grain boundaries, confirms that the basic assumptions of the ALAMEL model are qualitatively correct, except at triple junctions. Splitting of one of the grains was occasionally observed, which has also been observed experimentally.
\end{abstract}

\section{Introduction}

Plastic deformation in metals is heterogeneous in nature. The primary reason for this is the requirement of stress equilibrium and strain compatibility at grain boundaries, triple junctions and quadruple junctions. Statistical models which make simple assumptions about the distribution of stresses and strains (such as the Taylor model) cannot capture these effects, resulting in poor predictions of deformation textures and imperfect predictions of plastic anisotropy. It has been found that slightly more sophisticated statistical models for the interactions between neighbouring grains improve the quality of these predictions very much [1]. One of these models is the Advanced Lamel (ALAMEL) Model [1]. In principle crystal plasticity finite element model (CPFEM) are even better at capturing plastic strain heterogeneities, but they require huge calculation times compared to statistical models. Nevertheless, the present study aims at using a CPFEM model to do a more in-depth assessment of the assumptions used by the ALAMEL concerning grain interactions.

\section{Short Description of the Advanced Lamel (ALAMEL) Model}

Most models for the plastic deformation of polycrystalline materials including the prediction of deformation textures assume that plastic deformation is achieved by means of crystallographic slip. For fcc metals, $\{111\}<110>$ slip systems are adopted, and for bcc metals, $\{110\}<111>+$ $\{112\}<111>$ systems. They all explicitly or implicitly adopt the generalised Schmid law (or its visco-plastic approximation) which states that a slip system is active when the resolved shear stress reaches a critical level, the 'critical resolved shear stress'. In many deformation texture models, the value of the latter has no effect on the result, so it may be set to be equal to a constant. However, some of the texture models let it increase as deformation goes on (work hardening). Fig. 1 illustrates 


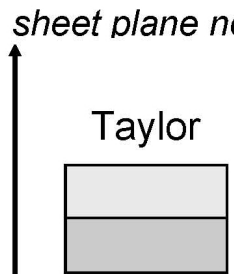

(a)

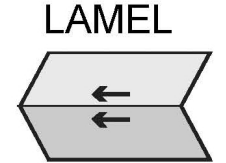

(b)

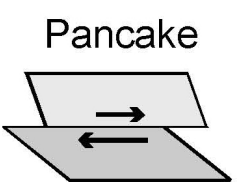

(c)

rolling direction

Fig.1 Hypothetical set of two grains taken from the mid-plane section of a sheet after cold rolling, according to several statistical models. The grains were had a cubic shape before rolling. (a) Taylor full constraints model. (b) LAMEL model. (c) Pancake Relaxed Constraints model.

three models: the full constraints Taylor model (FC Taylor), the Lamel model and the Pancake Relaxed Constraints model (RC Pancake) [1]. They are statistical models, which means that they do not take the details of the microstructure into account. Instead, they treat the crystals one by one, or in small clusters, and they take the crystal orientations in a statistical way from an orientation distribution function. Fig. 1 shows a hypothetical set of two grains taken from the mid-plane section of a rolled sheet. The crystal orientations are chosen at random from the discrete sets describing the current texture. The interface between the grains is parallel to the rolling plane. The grains had supposedly a cubic shape before rolling. According to the full constraints Taylor model [1-3], the two grains have still the same shape after rolling, corresponding to the basic assumption of the FC Taylor theory, namely that the plastic strain of all individual grains is equal to the average plastic strain of the polycrystal (Fig. 1a). According to the LAMEL model [1], the average shape change of a set of two stacked grains is still equal to the average shape change of the polycrystal, but in each of the two grains, shears have been allowed to take place (Fig. 1b), which are referred to as 'relaxations'. The shears in one grain are opposite to those in the other grain. Fig. 2 shows the type of relaxations allowed. The Pancake Relaxed Constraints model [1] is similar (fig. 1c), but it does not require that the relaxations in each of the grains are opposite.
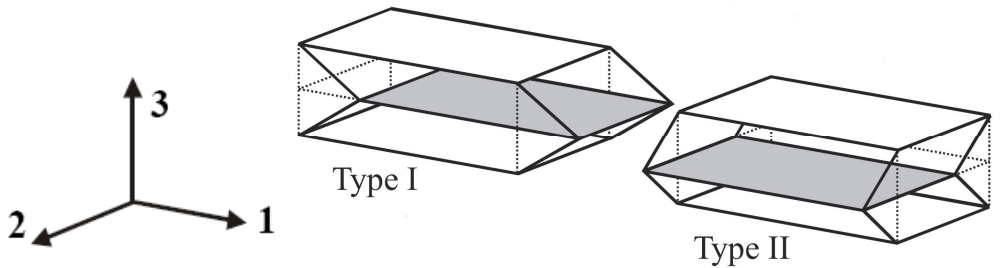

Fig. 2 Illustration of the two types of relaxation considered in the LAMEL model. Type I corresponds to RD-ND shear and Type II to TD-ND shear.

These models are used to identify the active slip systems for a given increment of macroscopic strain, find the slips and also (in case of the LAMEL model and the Pancake Relaxed Constraints model) the relaxations. For this, kinematical equations are used relating the slip activity and the relaxations to the macroscopic strain, as well as a condition stating that the plastic work dissipated in the grain or in the 2-grain cluster should be minimised. This is equivalent to applying the generalised Schmid law [4-5]. Once the slips are known, the local stress deviator and the lattice rotations can be obtained. The latter are then used to simulate the change in deformation texture caused by the increment of macroscopic strain. Note that models like these usually neglect elastic strains. For the mathematical details, we refer to other papers [1]. Keeping all this in mind, and looking back on the basic assumptions of the three models presented above, it is seen that the FC Taylor model and the Pancake Relaxed Constraints model can be solved grain by grain, whereas the LAMEL models has to be solved simultaneously for a pair of grains simultaneously. In this, the 
individual orientations of both grains must be taken into account. The solutions found for the stress deviators respects the stress equilibrium along the boundary separating the two grains of the set, at least as far as the shear stresses are concerned [1]:

$$
\sigma_{13}^{\prime 1}=\sigma_{13}^{\prime 2} \quad \text { and } \quad \sigma_{23}^{\prime 1}=\sigma_{23}^{\prime 2}
$$

in which $x_{1}$ is the rolling direction, $x_{2}$ the transverse direction and $x_{3}$ the normal to the sheet (RD, TD ad ND in fig. 2, respectively). The LAMEL model is called an 'interaction' model, because it focuses on the interaction between two adjacent grains, each with a lattice orientation taken at random from the texture. It however neglects interactions at other grain boundaries. A physical justification has been proposed for cold rolled materials with flattened grains which are nearly parallel with the rolling plane [1]. The Advanced Lamel Model (ALAMEL) [1] is a further variant of the LAMEL model. Fig. 3 shows its principle. The slip rates are not estimated in the centre of a grain, as the FC Taylor model implicitly does, but rather at grain boundaries [1]. A set of grain boundary segments is randomly chosen from the microstructure (measured or assumed). The slip rates are then calculated in the regions at both sides of the grain boundary, and very close to it (Regions 1 and 2, fig. 4). In this, the stress equilibrium across the grain boundary segment is to be taken in to account. This can be achieved by using the same constitutive equations as in the LAMEL model. A difference with the latter model is that the grain boundary segments between two regions need not be parallel with the rolling plane. As strain goes on, the grain boundary segments tend to rotate towards the rolling plane. As a result, the predictions of the LAMEL and ALAMEL models are expected to converge at large thickness reductions in rolling. However, the ALAMEL model is not limited to rolling; it can also be used to simulate other deformation processes. But it is needed to verify whether patterns such as those illustrated in fig. 2 really exist at grain boundary segments in a polycrystal, and whether the shear stresses are in equilibrium along those segments (Eq. (1)). A CPFEM simulation has been carried out to that purpose. It will be described in the next paragraph.

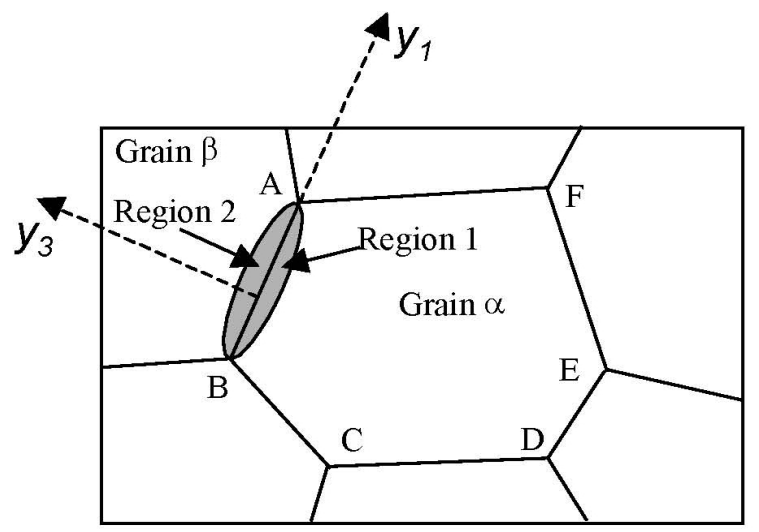

Fig. 3 Schematic representation of a microstructure as assumed by the ALAMEL model.

\section{Verification of the assumptions of the ALAMEL model though a CPFEM simulation}

A finite element mesh was constructed for a multicrystal consisting of four FCC grains with different crystal orientations. Each grain was discretised into a large number of elements (with 17496 integration points) to effectively capture the complex nature of the plastic fields in the vicinity of the grain boundaries. An elastic-viscoplastic constitutive law adapted to crystals deforming by dislocation slip was implemented in ABAQUS as user material (UMAT) module, the details of which have been given by Delannay et al. [6]. The material parameters used in this model have been described in detail by Kanjarla et al. [7]. The strain rate sensitivity exponent $m$ was given the value 0.012 , which is a reasonable value for a copper or an aluminium alloy (assuming absence of dynamic strain ageing) deforming at room temperature. The distribution of strain rates, both 
along and perpendicular to the grain boundaries, was analyzed in order to check the main assumptions of the ALAMEL model. These are:

(i) the velocity gradient tensors describing the ongoing deformation in a two boundary regions which are directly adjacent to each other along a grain boundary (Fig. 4b) differ from each other by opposite shear rates. The grain boundary is the shear plane.

(ii) the average velocity gradient tensors of a cluster consisting of the two boundary regions (Fig. 4a) is equal to the macroscopic velocity gradient tensor.

(iii) the shear stresses acting on the grain boundary are in equilibrium.

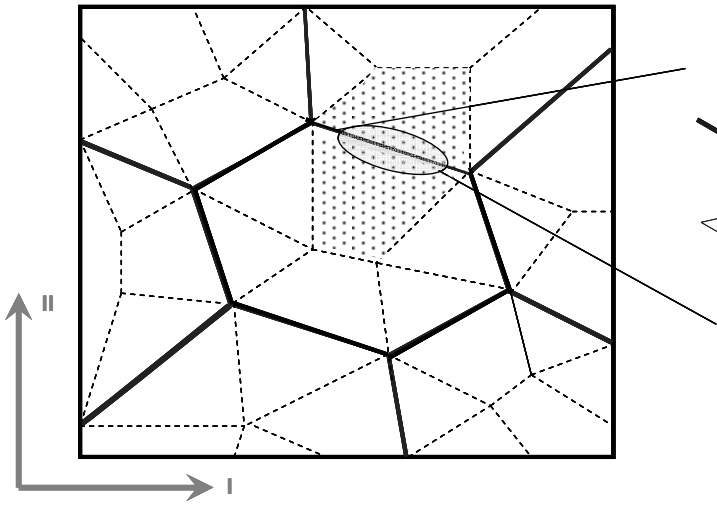

(a)

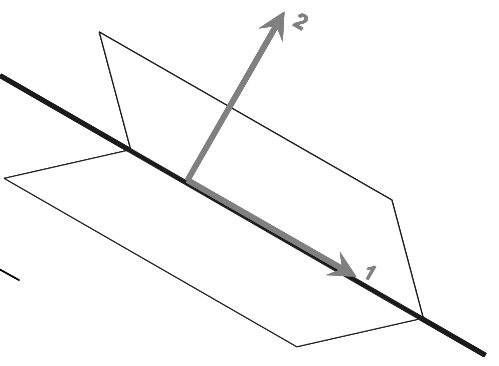

(b)

Fig. 4. (a) Schematic representation of the microstructure. Dark lines: grain boundaries. Shaded region: cluster as used in the ALAMEL model. (b) A Grain Boundary Segment (GBS) in a cluster, the reference frame attached to the GBS and the complementary shear relaxations.

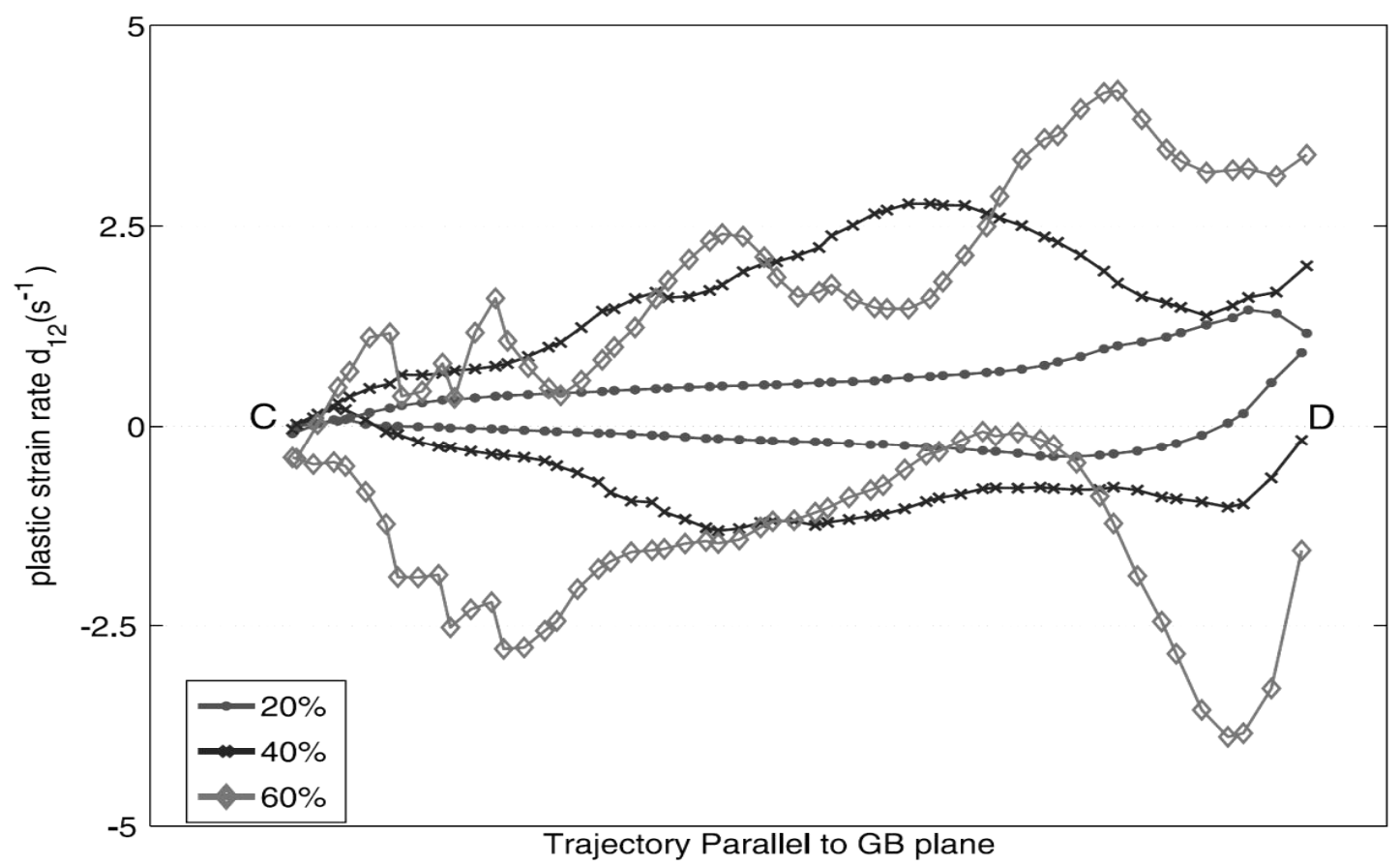

Fig. 5 Distribution of $d_{12}$ component of the local strain rate tensor, along the trajectory CD parallel to the grain boundary after Plane Strain Compression. C and D are triple junctions. CD is one of the nearly horizontal grain boundaries in Fig. 6. Legends indicate the thickness reduction in direction 2. (1-RD, 2-ND)

Generally speaking, the CPFEM simulations have shown that assumptions (i) and (iii) are correct, but assumption (ii) is not, i.e. the velocity gradient of the clusters is not equal to the average velocity gradient of the polycrystal. This is illustrated in fig. 5. It shows the evolution of the strain 
rate $d_{12}(\mathrm{RD}-\mathrm{ND})$ at a grain boundaries which is nearly normal to ND. For level of the macroscopic strain, the evolution of two values of $d_{12}$ is given, one for each of the two grains. It is seen that, at least at some distance of the triple junctions, these shears have indeed opposing signs, but they do not exactly compensate each other. Fig. 6 illustrates what has been observed at a triple junction. Grain 2 seems to split into two halves starting from the triple junction with grain 3 and grain 4 . At least in the vicinity of the triple junction, the boundary between grain 3 and grain 4 extends itself into grain 2, as do the opposing shears of the type shown in Fig. 4b. This suggests that at some triple junctions, a discrete shear strain heterogeneity of the type shown in Fig. 1 at the boundary of two of the three grains may extend into the third grain, thereby causing this grain to split after some strain (Fig. 7). Among all heterogeneous but geometrically admissible strain patterns that can be imagined at triple junctions, this one is clearly the simplest, but it requires the splitting of one of the three grains. It would be interesting to study whether this pattern corresponds to an energetically favourable solution for certain combinations of grain orientations. It can furthermore be seen in Fig. 6 that the strain in the upper half of grain 2 is very heterogeneous, which cannot be taken into account by a statistical model. Heavy oscillations of the stress and strain rate fields have furthermore been observed in the vicinity of the triple junction. They did not converge upon reductions of the mesh size. It is possible that at triple junctions, satisfactory results for detailed stress and strain rate distributions can only be obtained if the generalized Schmid law combined with a conventional hardening model (as done in our elastic-viscoplastic approach) is replaced by a model that implements the local dislocation interactions in a more precise way.

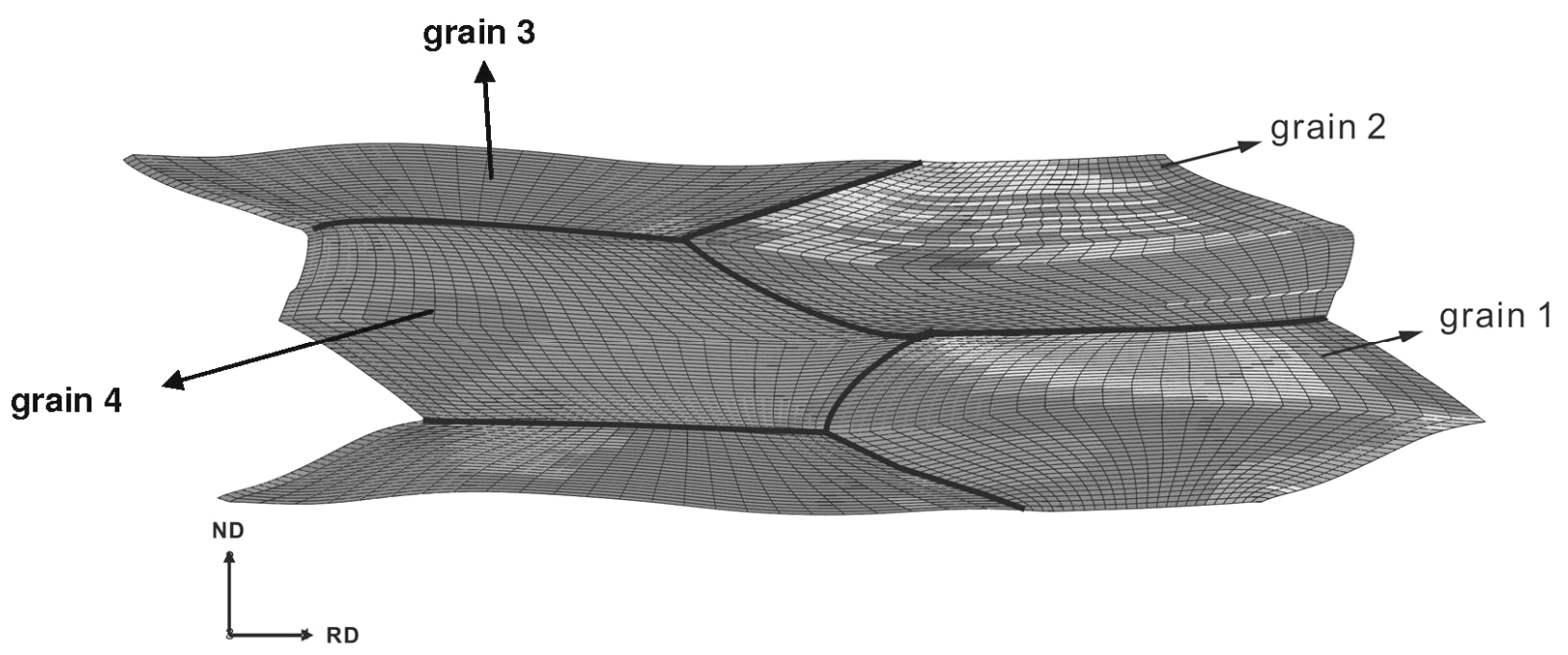

Fig. 6 Deformed mesh showing the model microstructure after $45 \%$ height reduction in plane strain compression. Gray scale: $d_{12}$ (RD-ND) component of the strain rate. See [7] for more details.

\section{Discussion and conclusion}

The main conclusion is that the average strain rate of the area of the two grains close to a grain boundary segment (shaded region in Fig. $4 \mathrm{a}$ ) is not equal to the macroscopic strain rate, although the deviation is not spectacular. A consequence of this is, that in the two grains touching each other at a grain boundary, the shear strain-type relaxations parallel to the grain boundaries indeed differ from each other, but they do not exactly compensate each other in order to make their average equal to the macroscopic shear strain rate. The corresponding shear stresses are in equilibrium at the grain boundaries. All this is true at some distance from the triple junctions. The CPFEM model has difficulty to find a solution for the stresses and strain rates close to the triple junctions. The model must probably be enriched by taking heterogeneous dislocation densities, lattice curvature, as well as the discrete nature of slip into account. 


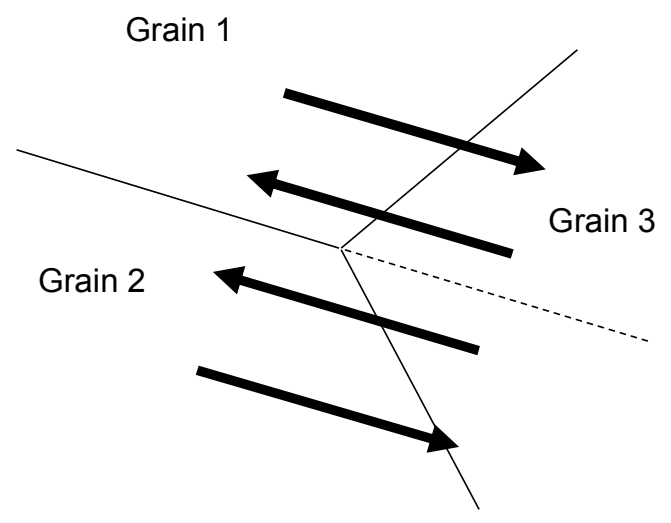

Fig 7 Hypothetical "simple" geometrically admissible strain pattern at a triple junction. A shear strain discontinuity as shown in fig. 1 exists at the boundary between grains 1 and 2 . The black arrows indicate the shear strain-type relaxations in these grains (deviations from homogeneous strain). The pattern extends itself in Grain 3, thereby causing the latter to split along the dashed line as strain goes on.

\section{Acknowledgements}

Funding from Belgian Science Policy through the grant IAP- 06/24 is gratefully acknowledged. Laurent Delannay is mandated by National Fund for Scientific Research (FNRS, Belgium).

\section{References}

[1] P. Van Houtte, S. Li, M. Seefeldt and L. Delannay: Int. J. Plast. Vol. (2005), p. 589

[2] G.I. Taylor: J. Inst. Metals Vol. 62 (1938) p. 307

[3] U.F. Kocks, C.N. Tomé, H.-R. Wenk: Texture and Anisotropy. Preferred Orientations in Polycrystals and their Effect on Material Properties. (Cambridge University Press, U.K. 1998).

[4] J.F.W. Bishop, R. Hill: Phil. Mag. Vol. 42 (1951) p. 414.

[5] J.F.W. Bishop, R. Hill: Phil. Mag. Vol. 42 (1951) p. 1298.

[6] L. Delannay, P.J. Jacques, S.R. Kalidindi: Int. J. Plast. Vol. 22 (2006), p. 1879.

[7] A.K. Kanjarla, P.Van Houtte and L. Delannay: Assessment of Plastic Heterogeneity in Grain Interaction Models using Crystal Plasticity Finite Element Method, submitted to Int. J. Plast. (2009) 